„Selbstentmachtungen gehen auf das Konto der Exekutive und häufig zu Lasten des Parlaments" (S. 48).

Der erfreulicherweise interdisziplinär gestaltete Sammelband ist in fünf Kapitel gegliedert: Der Staat der modernen Gesellschaft - Europäisierung der Staatlichkeit - Transformation von Wohlfahrtsstaatlichkeit - Politik und Öffentlichkeit im digitalen Zeitalter - Verfassungsstrukturen und Märkte. Der letzte Teil, eher randständig und in sich heterogen, zielt weniger auf die Frage nach der Souveränität des Staates. Die drei Beiträge zur Thematik „Europäisierung und Wandel der Staatlichkeit“ sind besonders aufschlussreich. Prinzipielle Überlegungen vermischen sich mit aktuellen Bestandsaufnahmen. Roland Sturm untersucht den Souveränitätstransfer von den Nationalstaaten zur EU, Enrico Peuker verweist auf die Europäisierung staatlicher Verwaltungen, Maurizio Bach analysiert kritisch die Rolle des Nationalstaates in der EU.

Für diesen Band gilt das Gesagte über Kielmanseggs Essaysammlung. Es mangelt etwas an Geschlossenheit. Sieht Kielmansegg im Nationalstaat eher einen Garanten der Demokratie, so nahmen manche Autoren in dem Sammelwerk gerade in Nationalstaaten postdemokratische Tendenzen wahr.

Eckhard Jesse

\title{
Das Bundesverfassungsgerichtsgesetz: kompakte und selbstbewusste Erläuterungen
}

\section{Lenz, Christofer und Ronald Hansel: Bundesverfassungsgerichtsgesetz. Handkommentar, Nomos} Verlagsgesellschaft, Baden-Baden 2013, 677 Seiten, € 98,-.

Der Markt für Kommentarliteratur zum Bundesverfassungsgerichtsgesetz ist relativ überschaubar: So gibt es das doppelbändige Loseblattwerk mit knapp 4.000 Seiten, das Theodor Maunz begründet hat und heute von ehemaligen Mitgliedern des Bundesverfassungsgerichts und Hochschullehrern betreut wird, sowie - bislang - die beiden Handkommentare von Hans Lechner und Rüdiger Zuck (6. Auflage 2010, 815 Seiten, $88 €$ ) sowie vom HerausgeberTeam Dieter C. Umbach, Thomas Clemens und Franz-Wilhelm Dollinger (2. Auflage 2005, 1.436 Seiten, $178 €$ ). Dieses Duo der Handkommentare bekommt nun Verstärkung und Konkurrenz durch das neu in erster Auflage erschienene Werk von Christofer Lenz und Ronald Hansel.

Angesichts der großen Bedeutung, die die Verfahren vor dem Bundesverfassungsgericht oftmals haben, ist es nur zu begrüßen, wenn sich das Angebot der dafür einschlägigen prozessrechtlichen Kommentarliteratur verbreitert. Gleichwohl stellt sich bei jedem Werk, das ein literarisch bereits erschlossenes Gebiet betritt, die Frage nach den Alleinstellungsmerkmalen und seinem wissenschaftlichen oder rechtspraktischen Mehrwert. In diesem Kontext ist zunächst einmal das Autorenduo selbst interessant: Während die beiden schon vorhandenen Kurzkommentare jeweils eine „geschlossene Perspektive“ aufweisen, nämlich die Bestimmungen des Verfassungsprozessrechts entweder aus der anwaltlichen Sicht (Lechner) Zuck) oder aus der Perspektive von wissenschaftlichen Mitarbeitern des Gerichts (Umbach) Clemens/Dollinger) erläutern, verbinden Lenz und Hansel diese beiden Perspektiven in einem Werk. Die anwaltliche „Außensicht“ auf das Verfassungsprozessrecht bringt Christofer Lenz ein, der seit 15 Jahren in einer bundesweit agierenden Anwaltskanzlei unter anderem 
für verfassungsprozessuale Fälle zuständig ist. Demgegenüber war der heute als Arbeitsrichter in Wuppertal tätige Ronald Hansel viele Jahre als wissenschaftlicher Mitarbeiter beim BVerfG tätig und bürgt damit für die Berücksichtigung der „Innensicht“ aus dem Karlsruher „Maschinenraum“. Um diese Verbindung der verschiedenen Sichtweisen durchgängig zu gewährleisten, haben die beiden Autoren das zu kommentierende Gesetz nicht untereinander aufgeteilt (wie dies bei vielen Zwei-Autoren-Werken der Fall ist), sondern das ganze Werk gemeinsam verfasst, wie im Vorwort betont wird.

Da beide forensische Praktiker sind, verwundert es nicht, dass der Kommentar stark auf diese Zielgruppe ausgerichtet ist. Besondere Auswirkungen hat das auf die Auswahl der berücksichtigten Judikatur des BVerfG. So haben Lenz/Hansel neben der Rechtsprechung der Senate auch diejenige der Kammern maßgeblich berücksichtigt und damit dem Umstand Rechnung getragen, dass mittlerweile ein Großteil der Verfahren durch die Kammern und nicht mehr durch die Senate erledigt wird (nominell fast 99 Prozent, vgl. \$15a Rn. 1). Zugleich konzentriert sich die Auswahl auf die Entscheidungspraxis seit 1999/2000, weil dieser nach Einschätzung der Autoren in der Praxis des Gerichts und seines gegenwärtigen Personals maßgebliche Bedeutung zukommt. Ebenfalls der Ausrichtung auf Praktiker ist die Fokussierung auf den aktuellen Gesetzestext unter weitgehendem Verzicht auf Hintergrundund Kontextinformationen geschuldet. Seiner Kommentarnatur gemäß ist das Werk daher weniger als lehrbuchhafte Erläuterung, sondern als Nachschlagewerk für den (mit einem gewissen Vorwissen ausgestatteten) Rechtspraktiker geeignet, der sich zur aktuellen Auslegung einer konkreten Bestimmung des BVerfGG kundig machen möchte. Die besondere Praxisorientierung zeigt sich schließlich an der Gewichtung der einzelnen Normen; so entfallen allein auf die Regelungen zur Verfassungsbeschwerde, die rund. 99 Prozent aller Verfahren ausmacht (vgl. die Fallzahlen von 2000 bis 2011 bei $\$ 13$ Rn. 6), 230 Seiten und damit rund ein Drittel des gesamten Werkes ( $\$ \$ 90-95$ BVerfGG).

Erfreulich ist, dass sich der Kommentar - zumal bei verfassungspolitisch umstrittenen Fragen - eine eigene Sichtweise und damit auch Kritikfähigkeit gegenüber dem BVerfG bewahrt, wie etwa an der kritischen Einordnung der Einbeziehung politischer Parteien in die Parteifähigkeit beim Organstreitverfahren ( $\$ 63$ BVerfGG) zu sehen ist. Ein weiteres Beispiel bietet die politisch und juristisch fragwürdige Wahl der vom Bundestag zu entsendenden Richter durch einen nichtöffentlich tagenden Wahlausschuss ( $\$ 6$ BVerfGG). Diese wird zwar in Übereinstimmung mit der Linie des Gerichts als zulässig verteidigt, aber mit einer dezidierten eigenen Bewertung, die auch vor einem kleinen Seitenhieb gegen Präsident Andreas Voßkuhle nicht zurückschreckt (dieser hatte die von ihm in einer GG-Kommentierung erhobene Forderung nach einer konkurrierenden Wahl in der Neuauflage nach seiner eigenen Wahl zum Bundesverfassungsrichter fallen gelassen, $\$ 6$ Rn. 3). Mit der Erläuterung des informellen Verfahrens der Kandidatenvorstellung im Vorfeld der Wahl widmet sich der Kommentar nicht nur den sich unmittelbar aus dem Gesetzestext ergebenden Rechtsfragen, sondern bezieht auch politisch-praktische Aspekte in die Darstellung ein ( $\$ 6 \mathrm{Rn}$. 9). Dies zeigt sich besonders bei den Ausführungen zum spannungsreichen Verhältnis zwischen der Normverwerfungskompetenz des Gerichts und der Gestaltungshoheit des Gesetzgebers. Hier setzen sich die Autoren intensiv und weitgehend kritisch mit den Tenorierungsvarianten auseinander, die das BVerfG bei als verfassungswidrig angesehenen Normen häufig anwendet. Dabei handelt es sich um die Unvereinbarkeitserklärung (mit inhaltlichen und zeitlichen Auflagen für den Gesetzgeber) und um die Übergangsregelungen (bei denen das Gericht unmittelbar als Ersatzgesetzgeber agiert) - vor dem Hintergrund, dass das BVerfGG 
bei verfassungswidrigen Normen eigentlich nur die Nichtigkeitsfolge kennt und keine positive Gestaltungsfunktion des BVerfG vorsieht (\$\$ 78 Rn. 13 ff., 95 Rn. 46 ff.).

Mit seiner politischen Sensibilität trägt das Werk dem besonderen Doppel-Charakter des Bundesverfassungsgerichts als justizförmiges Entscheidungsorgan und politischer Akteur in gelungener Weise Rechnung. Hinzu kommen eine klare Sprache und die eigenständige Positionierung in spannenden Streitfragen, was diesen Kommentar zu einer ebenso interessanten wie wertvollen Bereicherung der verfassungsprozessualen Literatur macht.

Volker M. Haug

\section{Das Bundesverfassungsgericht und die EU: Positionen auf dem Prüfstand}

van Ooyen, Robert Christian: Die Staatstheorie des Bundesverfassungsgerichts und Europa, 4. Auflage, Nomos Verlagsgesellschaft, Baden-Baden 2011, 236 Seiten, € 29,-.

Seit langem behandelt Robert Christian van Ooyen die rechtspolitische Stellung moderner Staaten, hier mit Blick auf die Europa-Rechtsprechung des Bundesverfassungsgerichts. Seit 1967 urteilt Karlsruhe über die Stellung des Europarechts. Vermied das Gericht noch im Urteil vom 30. Juli 1952 im Zusammenhang mit der Europäischen Verteidigungsgemeinschaft (BVerfGE 1, S. 396 ff.) Aussagen zur im EVG-Vertrag vorgesehenen Europäischen Politischen Gemeinschaft, befand es am 18. November 1967 im Votum zu den EWG-Verordnungen, das Gemeinschaftsrecht sei eine eigenständige Rechtsquelle (BVerfGE 22, S. 293 ff.), und folgte damit den EuGH-Urteilen vom 5. Februar 1963 in der Rechtssache van Gend \& Loos und vom 15. Juli 1964 in der Rechtssache Costa / ENEL zu dessen absolutem Vorrang. Dies ist der Ausgangspunkt der äußerst straffen Darstellung van Ooyens.

Der Autor beschreibt die relevanten Entscheidungen, wovon im Anhang große Teile oder die Leitsätze abgedruckt sind, leider ohne die Randnummern der Internet-Exemplare bzw. Seitenzahlen der Entscheidungssammlung. Ausführlich behandelt er die „Solange-Beschlüsse“: In „Solange I“ (BVerfGE 37, S. 271 ff.) konstatierte das BVerfG am 29. Mai 1974, mangels europäischen Grundrechtskatalogs könne europäisches Sekundärrecht, Rechtsakte, auch EuGH-Urteile am Grundgesetz überprüfen; dieser Vorbehalt wurde am 22. Oktober 1986 im „Solange II“-Beschluss zurückgestellt. Im Maastricht-Urteil vom 12. Oktober 1993 (BVerfGE 89, S. 155 ff.) ging das Gericht dann ob des vom Autor stringent dargestellten etatistischen Demokratiemodells um den souveränen Staat aus eigenem Recht und das Staatsvolk als homogene politische Einheit. Zugleich wurde damit Karlsruhes Anspruch auf „Augenhöhe“ mit dem EuGH, gegebenenfalls als sein Korrektor formuliert, während am 4. April 2013 Frankreichs Conseil constitutionnel - dem Bundesverfassungsgericht vergleichbar - in der Décision 2013-314P QPC eine Streitfrage dem EuGH vorlegte und Österreichs Verfassungsgericht am 4. Mai 2012 auf der Grundlage der EU-Grundrechtecharta urteilte; das BVerfG hingegen würdigte zur EGMR-Entscheidung in Sachen Görgülü die Europäische Menschenrechtskonvention zu bloß einfachem Recht unterhalb des Grundgesetzes herab (BVerfGE 111, S. 307 ff.). Im Urteil zur Antiterrordatei vom 24. April 2013 (1 BvR 1215/07) kommt es ohne konkreten Anlass zum EuGH-Urteil vom 26. Februar 2013 in der Finnland betreffenden Rechtssache Akerberg Fransson sogar zum Angriff: Im Sinne eines 